\title{
Multiscale Dynamics of Polymers in Particle-Rich Nanocomposites
}

Rahul Mangal, Yu-Ho Wen, Snehashis Choudhury, and Lynden A. Archer* Supporting Information

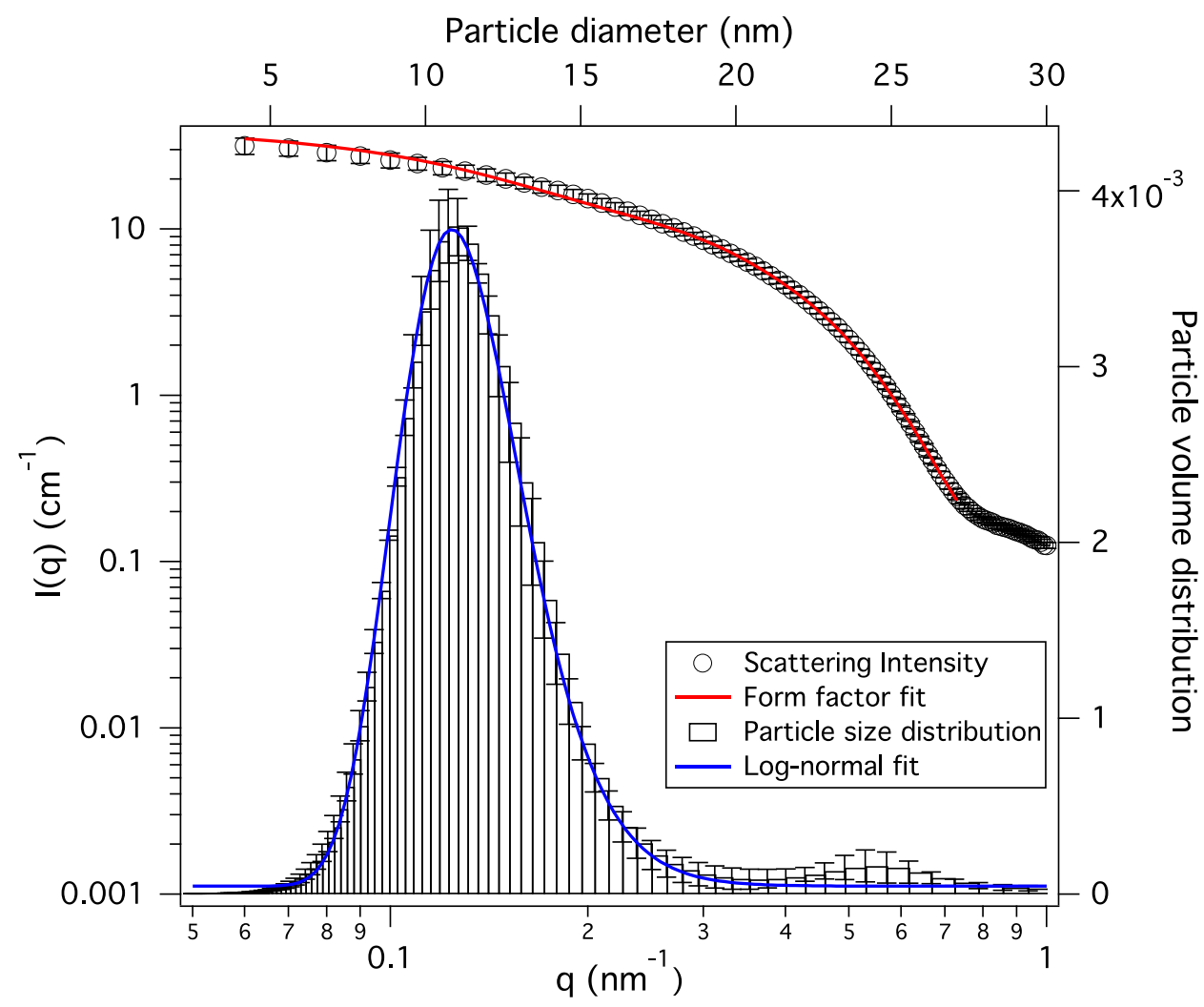

Figure 1S. Particle size distribution obtained from SAXS measurements for $0.95 \% \mathrm{w} / \mathrm{w}$ sulfonic acid functionalized silica nanoparticles in water. The fitting of the scattering intensity was carried out using the size distribution tool of the SAXS data-modeling package IRENA (http://usaxs.xray.aps.anl.gov/staff/ilavsky/irena.html).

The resultant particle size distribution was found to be well described by a log normal distribution, as shown in the blue line in the figure. Here $A, D_{\text {avg }}$ and $\sigma$ are an arbitrary pre-factor, the average diameter and the standard deviation in the particle diameter, respectively. The average particle diameter and the standard deviation in the particle diameters are found to be $10.8 \pm 0.008 \mathrm{~nm}$ and $0.3 \pm 0.001$, respectively, as determined from the fit. 

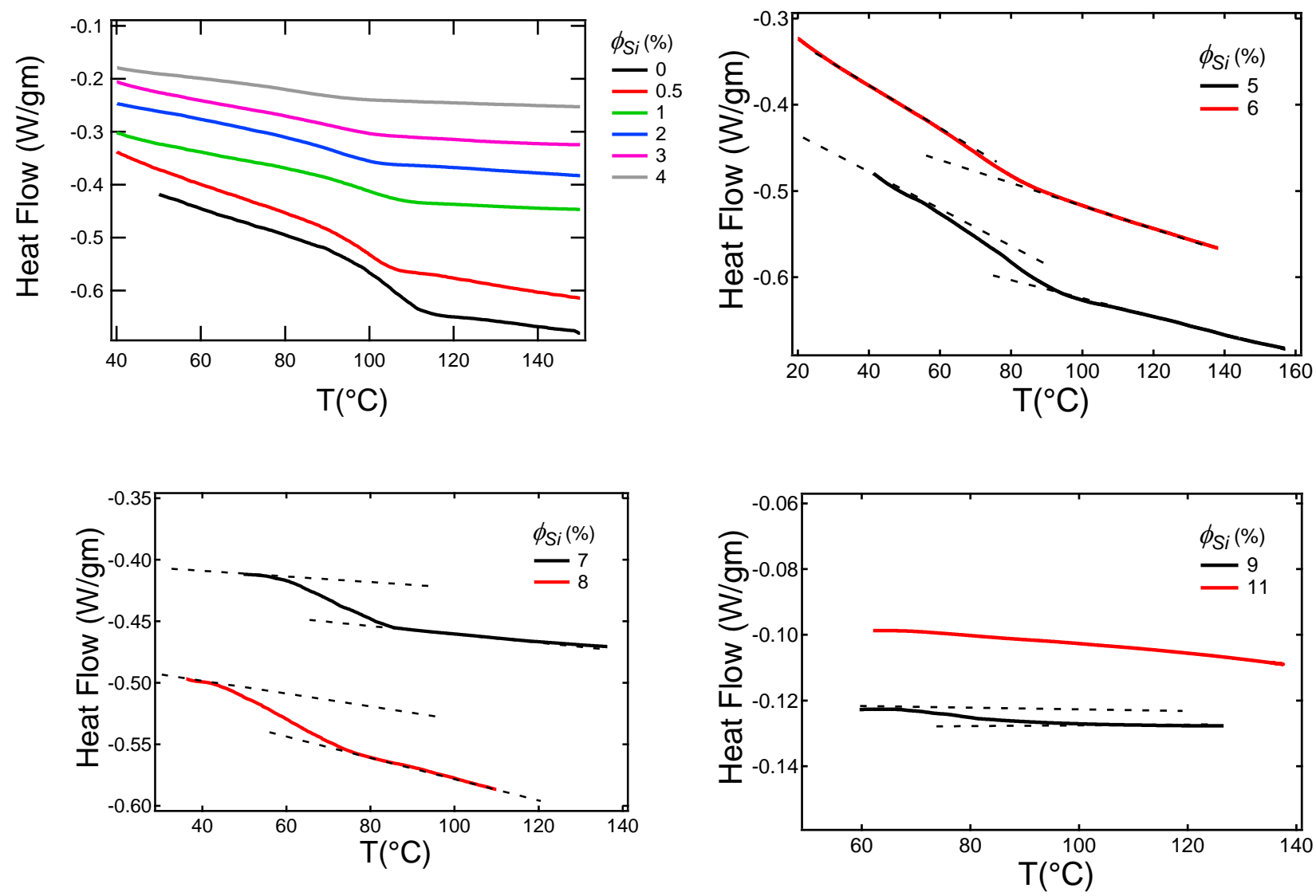

Figure 2S: Heat flow vs temperature from DSC measurements for $\mathrm{SiO}_{2}-\mathrm{PEG}_{2 k} / P M M A_{55 k} P N C s$.
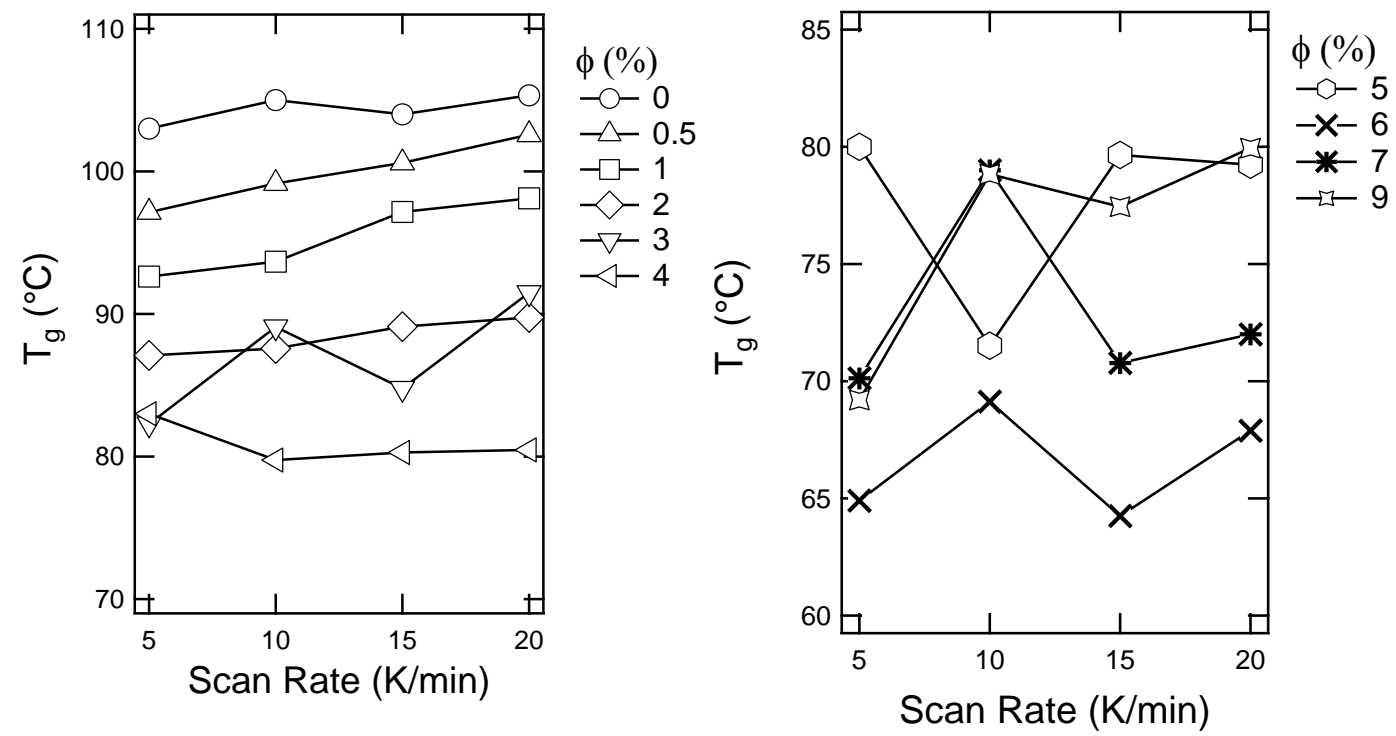

Figure 3S: Variation in Tg values obtained from DSC measurements at different scan rates $\mathrm{SiO}_{2}-$ $P E G_{2 k} / P M M A_{55 k} P N C s$. 


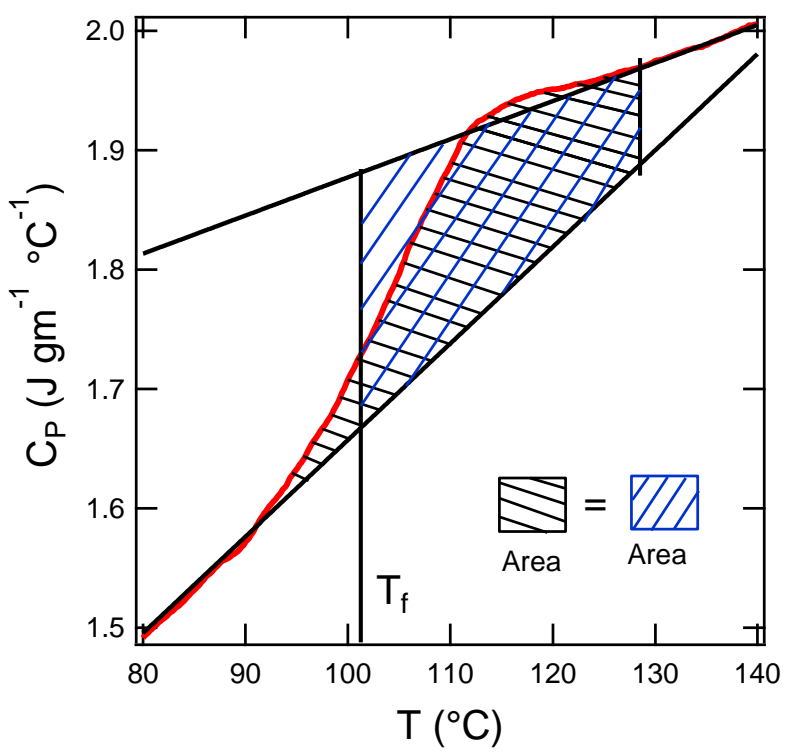

Figure 4S: Application of Moynihan's method to determine the fictive temperature, $T_{f}$, of neat PMMA 55k at scan rate of $20 \mathrm{~K} \mathrm{~min}^{-1}$. Here, the fictive temperature is defined as when the forwardslashed area is equal to the backward-slashed area.

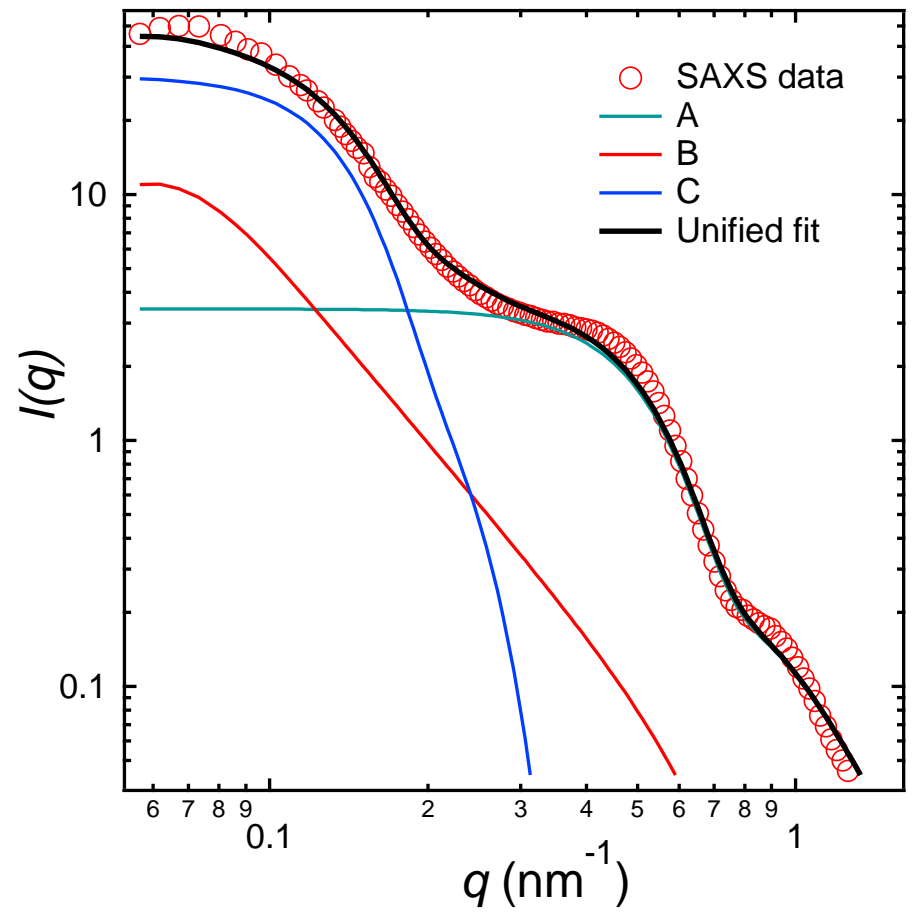

Figure 5S: $I(q)$ vs $q$ data obtained from SAXS measurements for $\mathrm{SiO}_{2}-P_{E G_{2 k}} / P M M A_{55 k} \phi=8 \%$. Black line is the Unified Beaucage fit where A corresponds to the Guinier term for the particle itself, $C$ is the Guinier fit to the correlated domains so size $\sim 65 \mathrm{~nm}$ and $B$ is the power law scattering from the correlated domains with power law $\sim 2$. 

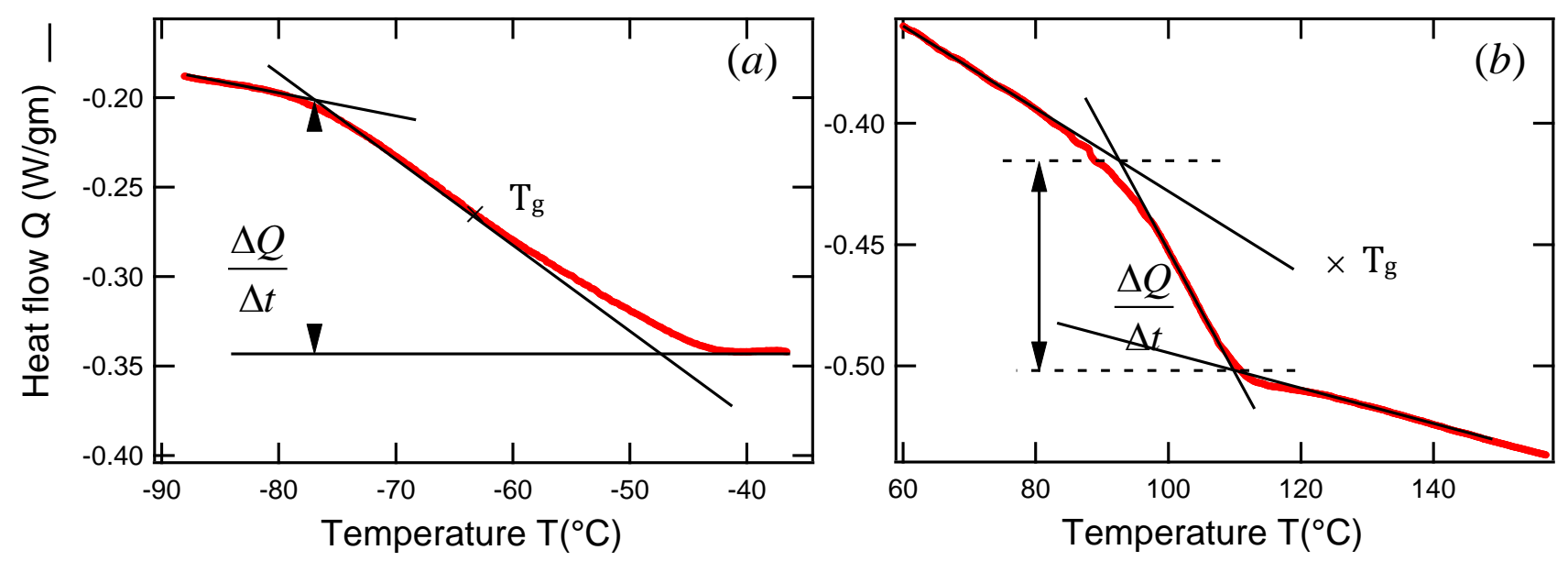

Figure 6S: Heat flow vs temperature data at $T_{g}$ for (a) $P E G_{2 k}(b) P M M A_{55 k}$ obtained from DSC measurements at ramp rate $\frac{\Delta T}{\Delta t}$ of $15 \mathrm{~K} / \mathrm{min}$. Change in heat capacity at $T_{g}$ was obtained using $\Delta c_{p}=\frac{\Delta Q}{\Delta t} \frac{\Delta t}{\Delta T}$

\begin{tabular}{|c|c|c|c|c|c|c|c|}
\hline Component & $\begin{array}{c}\boldsymbol{T}_{\boldsymbol{g}} \\
\left({ }^{\circ} \mathrm{C}\right) \\
\end{array}$ & $\begin{array}{c}\rho \\
(\mathrm{gm} / \mathrm{ml})\end{array}$ & $\begin{array}{c}\boldsymbol{M} \\
(\mathrm{gm} / \mathrm{mol}) \\
\end{array}$ & $\begin{array}{c}\Delta \boldsymbol{c}_{p} \\
(\mathrm{~J} / \mathrm{gmK})\end{array}$ & \multirow[t]{2}{*}{$k_{P E G-P M M A}$} & \multirow[t]{2}{*}{ APEG-PMMA } & \multirow[t]{2}{*}{$\chi_{P E G-P M M A}$} \\
\hline PEG2k & -60.3 & 1.01 & 44 & 0.34 & & & \\
\hline $55 \mathrm{k}$ & 104.4 & \multirow{2}{*}{1.18} & \multirow{2}{*}{100} & 0.30 & 0.88 & $-2.5 \pm 8$ & $-0.03 \pm 0.08$ \\
\hline $280 \mathrm{k}$ & 120.1 & & & 0.28 & 0.82 & $2.0 \pm 8$ & $0.029 \pm 0.05$ \\
\hline
\end{tabular}

Table 1S: Parameters from Lu-Weiss fit of the DSC measured $T_{g}$ data. 
(a)

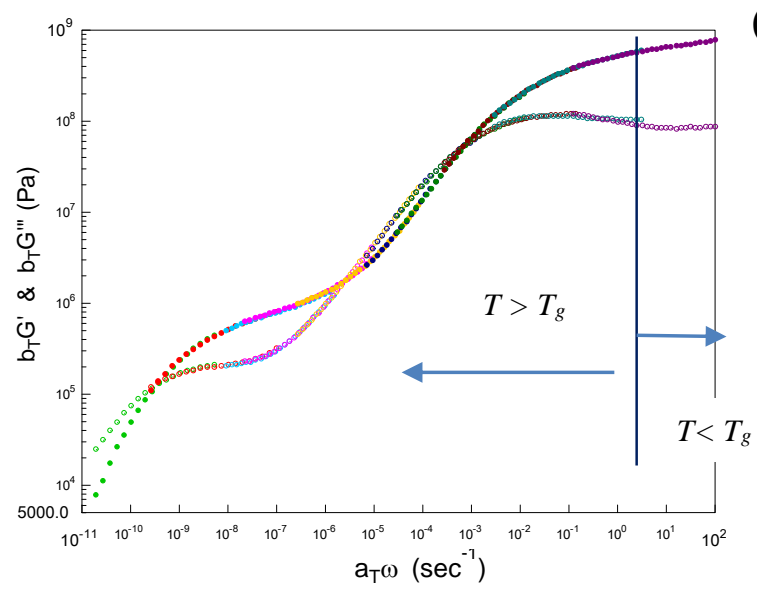

(c)

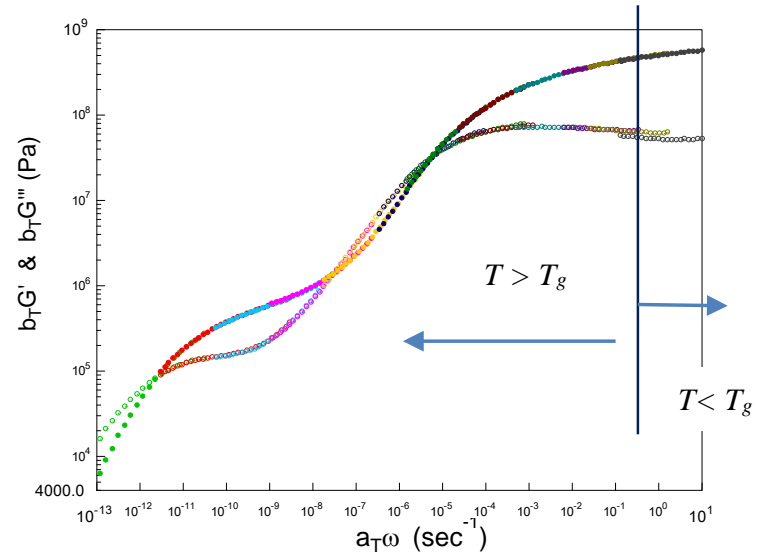

(b)

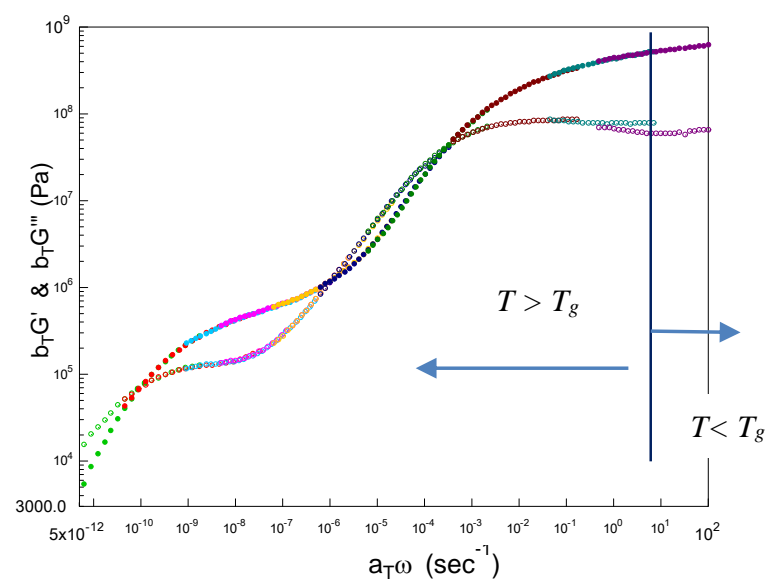

(d)

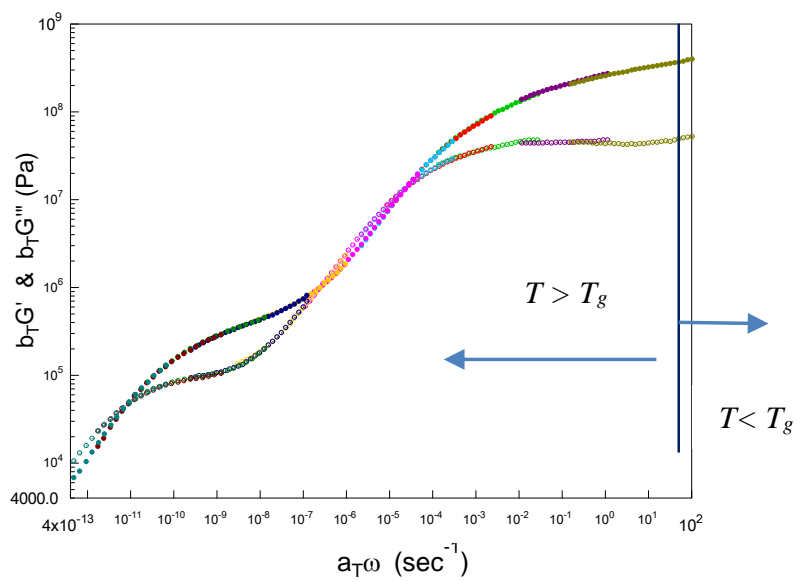

$(f)$

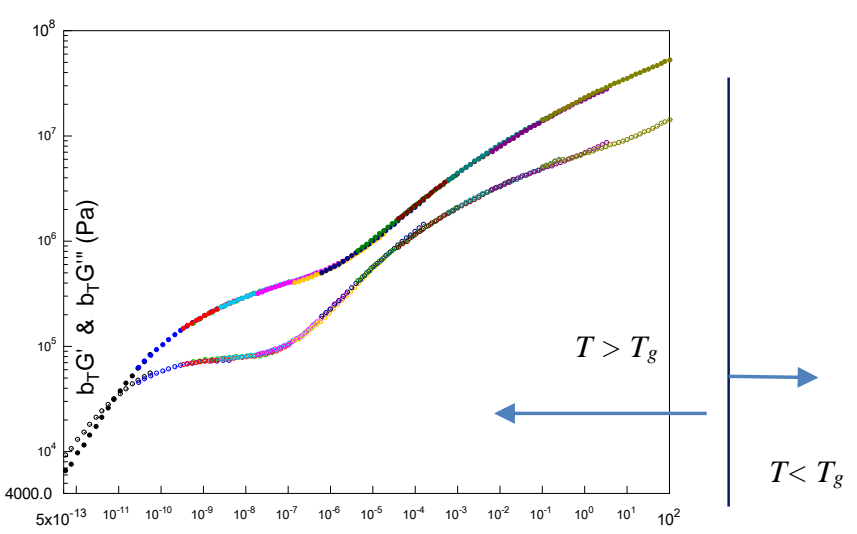

$a_{\top} \omega\left(\sec ^{-1}\right)$ (e)

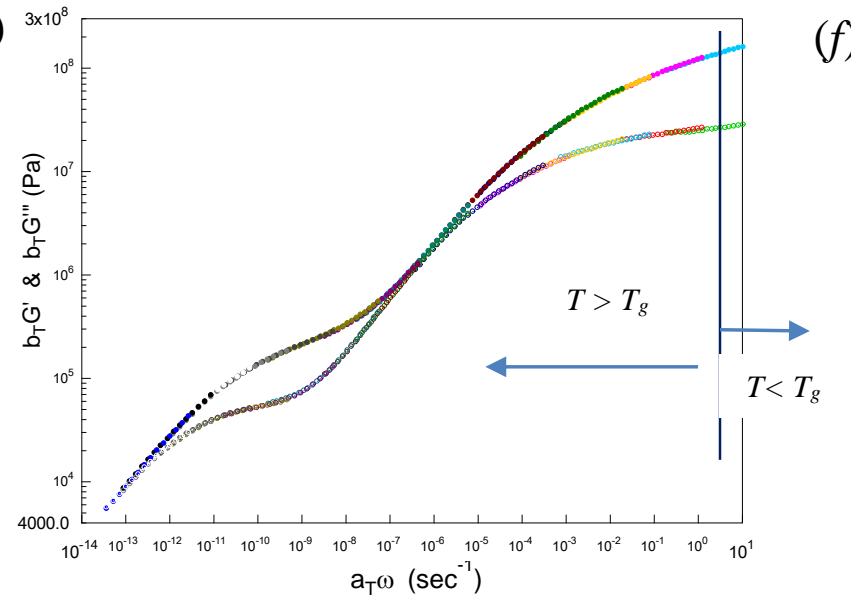

$(g)$

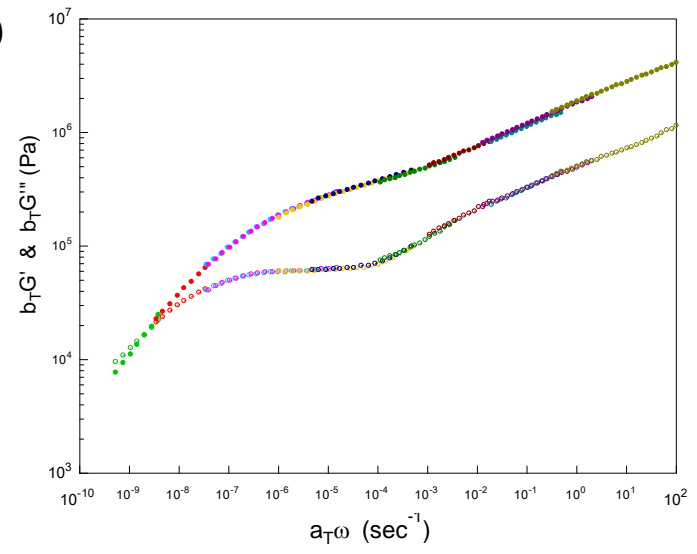

Figure 7S. TTS master curves with curves at different temperatures with different colors for $\mathrm{SiO}_{2}-\mathrm{PEG}_{2 k} / \mathrm{PMMA}_{55 k} \quad$ with (a) $\phi=0 \%$ (b) $\phi=1 \%$ (c) $\phi=2 \%(d) \phi=3 \%(e) \phi=5 \%(f) \phi=8 \%$ (g) $\phi=11 \%$. 


\begin{tabular}{|c|c|c|c|c|}
\hline \multirow[t]{2}{*}{$\phi(\%)$} & \multicolumn{2}{|c|}{$\begin{array}{c}\text { From } G^{\prime} \& G^{\prime \prime} \\
\text { Crossover }\end{array}$} & \multirow{2}{*}{$\begin{array}{c}\text { From tangent method } \\
\tau_{e}(\mathrm{sec})\end{array}$} & \multirow[t]{2}{*}{$\tau_{t}(\mathrm{sec})$} \\
\hline & $\tau_{o}(\mathrm{sec})$ & $\tau_{e}(\mathbf{s e c})$ & & \\
\hline 0 & $4.22 \times 10^{1}$ & $1.08 \times 10^{4}$ & $2.82 \times 10^{4}$ & $1.09 \times 10^{8}$ \\
\hline 0.25 & $9.37 \times 10^{1}$ & $2.45 \times 10^{4}$ & $4.86 \times 10^{4}$ & $2.43 \times 10^{8}$ \\
\hline 0.5 & $1.64 \times 10^{2}$ & $4.31 \times 10^{4}$ & $1.03 \times 10^{5}$ & $4.19 \times 10^{8}$ \\
\hline 0.75 & $1.72 \times 10^{3}$ & $4.96 \times 10^{5}$ & $1.16 \times 10^{6}$ & $5.45 \times 10^{9}$ \\
\hline 1 & $1.12 \times 10^{4}$ & $2.90 \times 10^{5}$ & $6.45 \times 10^{6}$ & $3.34 \times 10^{10}$ \\
\hline 1.25 & $1.85 \times 10^{4}$ & $4.27 \times 10^{5}$ & $9.01 \times 10^{5}$ & $5.02 \times 10^{9}$ \\
\hline 1.5 & $5.83 \times 10^{3}$ & $1.16 \times 10^{6}$ & $2.04 \times 10^{6}$ & $9.33 \times 10^{10}$ \\
\hline 2 & $3.13 \times 10^{4}$ & $5.60 \times 10^{6}$ & $1.17 \times 10^{7}$ & $6.41 \times 10^{10}$ \\
\hline 3 & $4.62 \times 10^{4}$ & $4.80 \times 10^{6}$ & $1.04 \times 10^{7}$ & $1.18 \times 10^{11}$ \\
\hline 4 & $9.88 \times 10^{4}$ & $3.08 \times 10^{6}$ & $1.49 \times 10^{7}$ & $4.54 \times 10^{11}$ \\
\hline 5 & NA & NA & $4.67 \times 10^{7}$ & $4.17 \times 10^{12}$ \\
\hline 6 & NA & NA & $6.25 \times 10^{6}$ & $4.59 \times 10^{11}$ \\
\hline 7 & NA & NA & $2.61 \times 10^{5}$ & $6.80 \times 10^{10}$ \\
\hline 8 & NA & NA & $2.77 \times 10^{4}$ & $2.99 \times 10^{9}$ \\
\hline 9 & NA & NA & $1.43 \times 10^{6}$ & $8.93 \times 10^{10}$ \\
\hline 11 & NA & NA & $5.92 \times 10^{2}$ & $1.27 \times 10^{8}$ \\
\hline 13 & NA & NA & $8.00 \times 10^{-1}$ & $1.34 \times 10^{7}$ \\
\hline 15 & NA & NA & NA & NA \\
\hline 16 & NA & NA & NA & NA \\
\hline
\end{tabular}

NA: not applicable

Table 2S. Segmental relaxation time $\left(\tau_{o}\right)$, entanglement relaxation time $\left(\tau_{e}\right)$ and the terminal relaxation time $\left(\tau_{t}\right)$ obtained from the TTS master curves shown in Figure 4. 


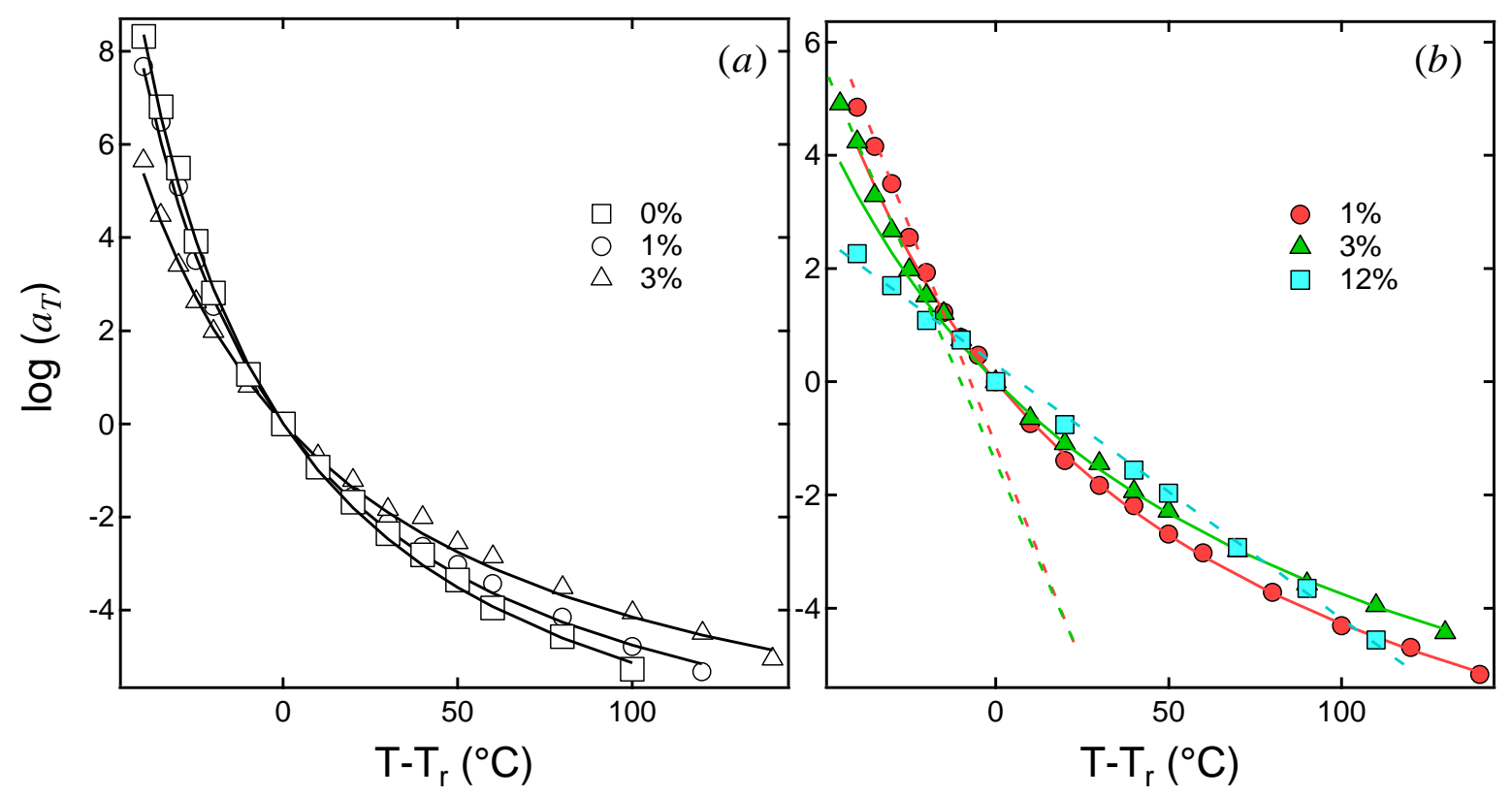

Figure 8S: Horizontal shift factor $\left(a_{T}\right)$ vs. temperature difference $\left(T-T_{r}\right)$ for $\mathrm{SiO}_{2}-P E G_{2 k} / P M M A_{280 k}$. In (a) solid line represents the corresponding WLF fit and in (b) the dashed lines represent the Arrhenius fit.

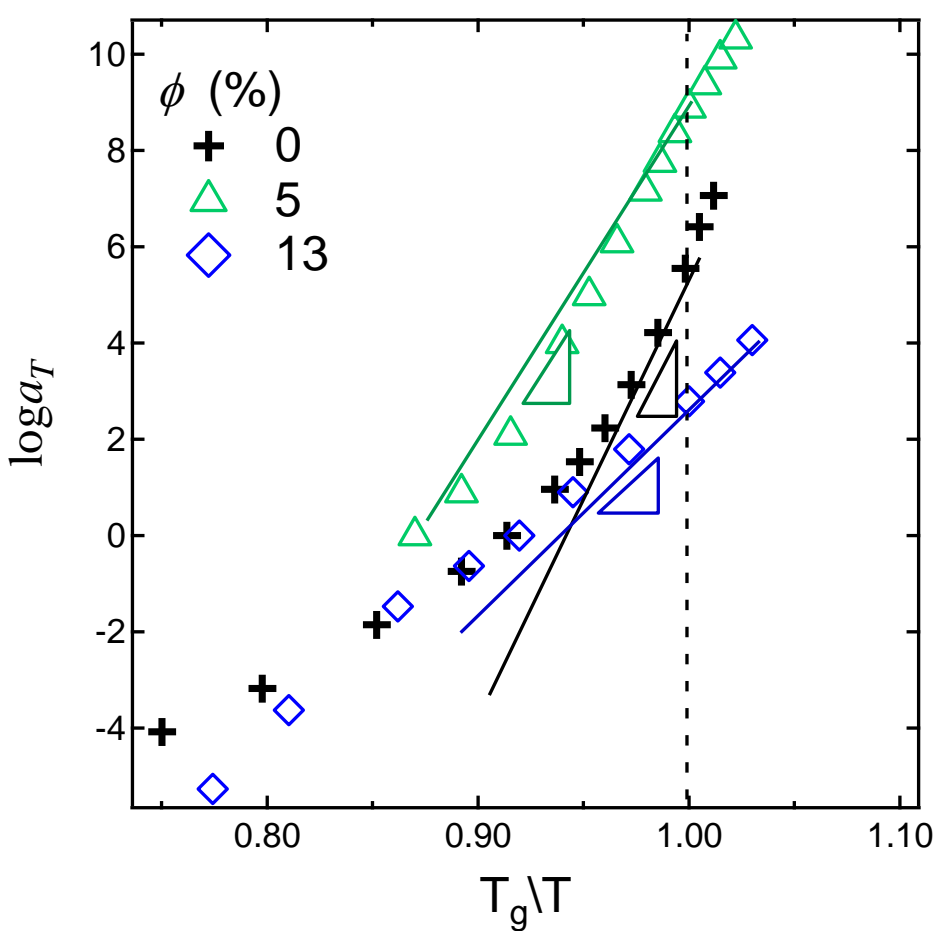

Figure 9S. $\log a_{T}$ vs $T_{g} / T$ for $\mathrm{SiO}_{2}-P E G_{2 k} / P M M A_{55 k}$. Solid lines denote the tangent at $T=T_{g}$. The slope of the tangent gives the fragility index $m$. 